\title{
Are Inter-Beat Intervals From Blood Pressure a Valid Alternative to R-R Intervals for the Multiscale Entropy Analysis of Heart Rate Variability?
}

\author{
Andrea Faini ${ }^{1}$, Gianfranco Parati $^{1}$, Paolo Castiglioni ${ }^{2}$ \\ ${ }^{1}$ Istituto Auxologico Italiano, IRCCS, Department of Cardiovascular, Neural and Metabolic Sciences, \\ S. Luca Hospital, Milan, Italy \\ ${ }^{2}$ IRCCS Fondazione Don Carlo Gnocchi, Milan, Italy
}

\begin{abstract}
When an ECG recording is not available to derive the $R-R$ interval (RRI) series, the heart rate variability $(H R V)$ may be evaluated considering the inter-beat intervals (IBI) from other cardiovascular signals, like the arterial blood pressure $(A B P)$. However, it is unknown how the $A B P$-derived IBI series quantify the multiscale entropy (MSE) of HRV. Thus our aim is to describe differences between RRI and the ABP-derived IBI in MSE estimates. We recorded the ECG and the finger $A B P$ in 40 volunteers. We derived the RRI series from the ECG (reference IBI) and the series of systolic-systolic intervals (SSI), diastolic-diastolic intervals (DDI), and intervals between maxima of the first- (d'PI) and second- (d"PI) derivative of $A B P$. For each IBI series, we estimated the $M S E$ at the scale $s=1$ beat (SampEn), at high-frequency scales $\left(M S E_{H F}\right.$, for $2 \leq s \leq 7$ beats) and low-frequency scales $\left(M S E_{L F}\right.$, for $7<s \leq 25$ beats), comparing estimates from the ABP-derived IBI with the RRI reference.

Sampen was substantially overestimated while $M S E_{H F}$ and $M S E_{L F}$ were underestimated by $A B P$-derived IBI. The estimates closest to RRI were provided by d'PI (error $+11 \%$ for SampEn, $-2 \%$ for MSE $E_{H F}$ ) and SSI (-2\% for $\left.M S E_{L F}\right)$. Thus, the ABP-derived IBI series reflect SampEn components absent in RRI, being d'PI and SSI the closest surrogate for RRI.
\end{abstract}

\section{Introduction}

Many studies of heart rate variability (HRV), in humans or animal models, are based on experimental setups that do not include the recording of an ECG tracing. In these cases, the heart rate is alternatively evaluated from the series of inter-beat intervals (IBI) derived from another cardiovascular signal, typically the arterial blood pressure (ABP) that in humans can be easily measured noninvasively at the finger site. Technical and methodological aspects for deriving the IBI series from $\mathrm{ABP}$ as well as physiological factors related to the origin of the ABP pulse may be responsible for differences in some HRV indices in comparison to measures from the $\mathrm{R}-\mathrm{R}$ intervals (RRI) of the ECG. For instance, the time of occurrence of the start of each cardiac beat is usually identified less precisely from the foot of the ABP waveform than from the R-peak of the ECG. Furthermore, factors influencing the transmission of the $\mathrm{ABP}$ wave along the arteries may also play a role. In this regard, the high-frequency spectral power is greater when measured from the ABP pulse rate detected at the finger than from the ECG heart rate, the difference being probably due to respiratory modulations of the pulse wave velocity [1].

Among the more recent HRV methods, the Multiscale Entropy (MSE) is arousing increasing interest because it was specifically designed to detect alterations in the cardiovascular complexity [2]. However, it is still unknown whether the IBI series derived from $\mathrm{ABP}$ can be used as surrogates of the R-R intervals (RRI) of the ECG to quantify the MSE components. It is also unknown whether there are methods for deriving the IBI series from the ABP that estimate MSE better than others. Aim of this work is therefore to compare MSE estimated from different ABP-derived IBI series with the reference MSE estimated from the RRI series.

\section{Methods}

We recorded the ECG and the finger ABP at $200 \mathrm{~Hz}$ during 15 minutes of supine rest in 40 healthy participants (19 women and 21 men, mean age \pm SD of $36 \pm 8.6$ years). Each R peak of the ECG was identified by a derivativeand-threshold algorithm using the parabolic interpolation to refine the position of the $\mathrm{R}$ peak. We identified the position of the corresponding diastolic and systolic pressure on the ABP signal, and of the maximum of the first and second ABP derivative, obtaining the IBI series of systolic-systolic intervals (SSI), diastolic-diastolic intervals (DDI), and of the intervals between maxima of the first (d'PI) and second ABP derivative (d"PI), as shown in figure 1. 

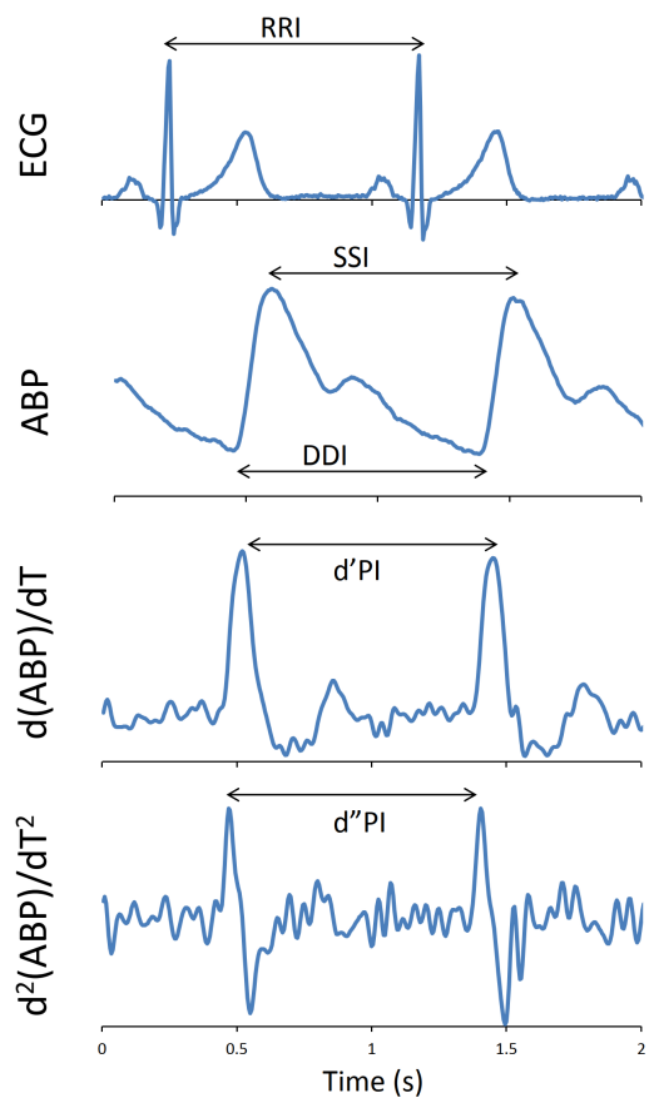

Figure 1. Definition of the ABP-derived IBI series: DDI=diastolic-diastolic intervals; SSI=systolicsystolic intervals; d'PI=intervals between maxima of the ABP first derivative; $\mathrm{d} " \mathrm{PI}=$ intervals between maxima of the ABP second derivative; RRI is the reference IBI from the ECG.

For each IBI series we calculated the MSE as in [3], employing a Butterworth filter for coarse graining, with embedding dimensions $m=1$ and $m=2$, setting the tolerance to $20 \%$ the standard deviation of the series and applying a fixed-tolerance strategy [4]. The algorithm provided MSE coefficients for scales $s$ between 1 beat (i.e., Sample Entropy, SampEn) and 50 beats. We also calculated a short-term MSE index, MSE $\mathrm{HF}_{\mathrm{HF}}$ averaging $\operatorname{MSE}(s)$ over $2 \leq s \leq 7$ beats, and a long-term MSE index, $\mathrm{MSE}_{\mathrm{LF}}$, averaging $\operatorname{MSE}(s)$ over $7<s \leq 25$ beats (these ranges of scales approximately correspond to the highfrequency and low-frequency components of spectral analysis, respectively).

The RRI was the reference IBI. The MSE estimates using SSI, DDI, d'PI or d"PI were compared with the reference estimates by the Wilcoxon signed-rank test.

\section{Results}

Figure 2 shows the profiles of $\operatorname{MSE}(s)$ for the ABPderived IBI series and the reference RRI. At $s$ equal to 1
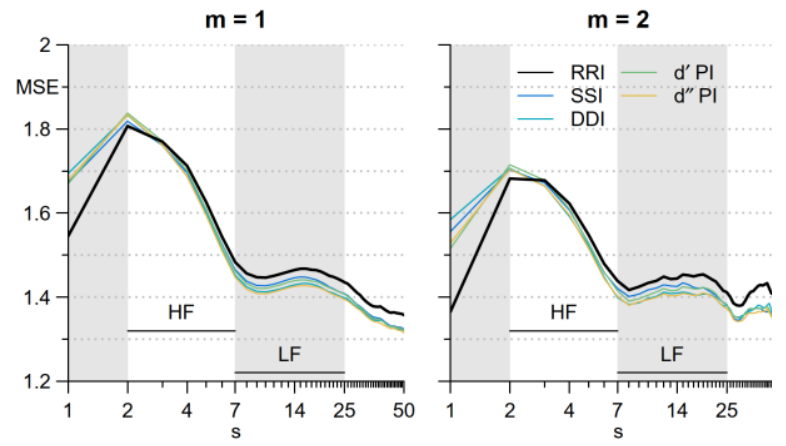

Figure 2. Multiscale entropy MSE (mean over 40 participants) for embedding dimensions 1 and 2 . The black lines are the reference estimates from RRI, the colour lines are estimates from ABP-derived IBI. The horizontal bands show the scale ranges used for calculating $\mathrm{MSE}_{\mathrm{HF}}$ and $\mathrm{MSE}_{\mathrm{LF}}$.

and 2 beats, MSE is substantially overestimated by all the ABP-derived series. The overestimation disappears at $s=3$ beats and at larger scales the ABP-derived IBI series underestimate the reference MSE.

Table 1 reports the values of the MSE indices for each type of IBI series. The SampEn overestimation by the ABP-derived IBI series as well as the $\mathrm{MSE}_{\mathrm{HF}}$ and $\mathrm{MSE}_{\mathrm{LF}}$ underestimations are statistically significant.

Table 1. Mean (SD) of MSE indices for different IBI.

\begin{tabular}{lcc}
\hline $\begin{array}{c}\text { Embedding } \\
\text { Dimension }\end{array}$ & $m=1$ & $m=2$ \\
\hline SampEn & & \\
$R R I$ & $1.544(0.270)$ & $1.365(0.272)$ \\
$S S I$ & $1.672(0.226) * *$ & $1.556(0.236) * *$ \\
$D D I$ & $1.694(0.222) * *$ & $1.584(0.225) * *$ \\
$d^{\prime} P I$ & $1.669(0.240) * *$ & $1.516(0.242) * *$ \\
$d^{\prime \prime} P I$ & $1.678(0.236) * *$ & $1.529(0.260) * *$ \\
MSE $_{\mathrm{HF}}^{*}$ & & \\
$R R I$ & $1.657(0.190)$ & $1.575(0.225)$ \\
$S S I$ & $1.646(0.187) * *$ & $1.565(0.221) * *$ \\
$D D I$ & $1.641(0.180) * *$ & $1.555(0.212) * *$ \\
$d^{\prime} P I$ & $1.651(0.185) * *$ & $1.567(0.220) * *$ \\
$d^{\prime \prime} P I$ & $1.639(0.202) * *$ & $1.554(0.233) * *$ \\
MSE $_{\mathrm{LF}}^{*}$ & & \\
$R R I$ & $1.455(0.239)$ & $1.438(0.261)$ \\
$S S I$ & $1.432(0.239) * *$ & $1.414(0.260) * *$ \\
$D D I$ & $1.418(0.236) * *$ & $1.400(0.259) * *$ \\
$d^{\prime} P I$ & $1.427(0.238) * *$ & $1.410(0.262) * *$ \\
$d^{\prime \prime} P I$ & $1.415(0.253) * *$ & $1.398(0.275) * *$ \\
\hline$* *$ means significantly different from RRI at $\mathrm{p}<0.01$
\end{tabular}

The amplitude of the discrepancies between the estimates by the ABP-derived IBI series and the reference estimate by RRI depends on the embedding dimension, the scale, and the type of IBI series, as figure 3 shows 

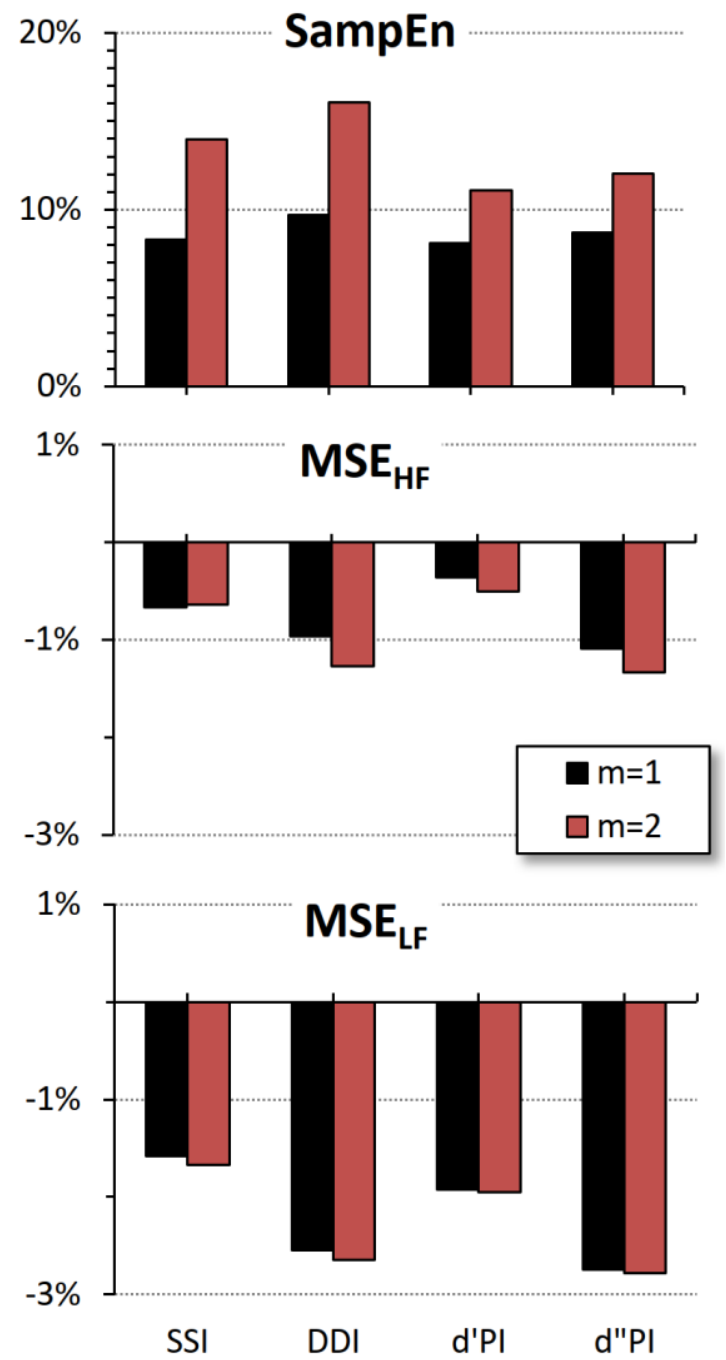

Figure 3. Discrepancies in MSE indices estimated by ABP-derived IBI series (see abbreviations in figure 1): the reference estimates are calculated from the RRI series. Values are shown as the mean percent error over $\mathrm{N}=40$ participants separately for embedding dimensions $m=1$ and $m=2$.

plotting the difference with the reference estimate expressed as the percent error of the estimate.

The largest discrepancies regard SampEn. With $m=1$ the overestimation is on average between $+8 \%$ (for SSI and $\mathrm{d}$ 'PI) and $+10 \%$ (for DDI); with $m=2$ the overestimation is even larger, ranging between $+11 \%$ (for d'PI) and $+16 \%$ (for DDI).

Percent errors are negative for $\mathrm{MSE}_{\mathrm{HF}}$ and $\mathrm{MSE}_{\mathrm{LF}}$, being these indices underestimated by the ABP-derived series. The absolute values of the error are lower for $\mathrm{MSE}_{\mathrm{HF}}$ and the estimates closest to the reference are obtained using d'PI (-0.4\% for $m=1,-0.5 \%$ for $m=2)$ and SSI $(-0.7 \%$ and $-0.6 \%$ for $m=1$ and $m=2)$. As to $\mathrm{MSE}_{\mathrm{LF}}$, percent errors are larger but again the estimates closest to the reference are those of SSI $(-1.6 \%$ and $-1.7 \%$ for $m=1$ and $m=2)$ and d'PI $(-1.9 \%)$. Differently from SampEn, the discrepancies in the $\mathrm{MSE}_{\mathrm{HF}}$ and $\mathrm{MSE}_{\mathrm{LF}}$ indices do not seem to depend on the embedding dimension.

\section{Conclusions}

This work demonstrates that the MSE estimates differ significantly when the HRV is assessed from IBI series derived from the finger ABP rather than from the RRI of the ECG. It also shows that the difference consists in a substantial overestimation at the scale of 1 beat and in an underestimation at scales larger than 3 beats. Considering the overall performance of the ABP-derived IBI series, we may conclude that the MSE estimates closer to the estimates provided by RRI are those obtained by using the d'PI or SSI beat-by-beat series.

The overestimation of SampEn by the ABP-derived IBI series could be explained by two factors. One could be the lower precision by which features of the ABP waveform define the time of occurrence of each cardiac beat, compared with the R peak of the ECG. Another factor could be the possible presence of specific noise components in the ABP signal, for instance, due to peripheral reflections of the blood pressure waves or to movements artifacts. We might assume that these two factors affect the IBI series with a mostly uncorrelated random noise. White-noise components superimposed to the ABP-derived series additively contribute to the signal entropy increasing the MSE estimates at the very short scales, a possible explanation of the overestimation we actually observed at the shorter scales. Since we applied a fixed-tolerance strategy, we expect that the effects of an additive white noise quickly vanish when the scales increase [4]: coherently, we did not detect any overestimation at scales greater than 2 beats. It should be noted that if we had applied a varying-tolerance approach, which is an MSE estimation strategy preferred by other authors [5], we could have found different results, with white-noise components affecting larger scales and likely causing overestimations also at $s>3$ beats.

An unexpected result is that all the ABP-derived IBI series significantly underestimated the MSE indices at scales greater than 3 beats, and particularly at the scales falling within the low-frequency range. This result indicates that at these scales the heart rate fluctuations observed from the peripheral pulse appear more predictable than those directly measured from the ECG. A possible interpretation is that the HRV quantified from the peripheral pulse rate includes regular and predictable components caused by systematic modulations of the pulse wave velocity. Modulations of the pulse wave velocity may be induced by the Mayer waves: this intrinsic oscillation of the cardiovascular system could have played a role in determining the MSE underestimation we observed. 
Finally, it should be noted that a recent study on freelymoving rats reported an overestimation of SampEn when calculated from the SSI or DDI series measured invasively into the femoral artery, rather than by the RRI series [6], a result similar to what we found in our work. However, DDI and not SSI provided the MSE estimates closer to the estimates calculated from RRI. By contrast, in our study the DDI series were associated with the largest overestimation of SampEn. This opposite conclusion suggests that the quantifications of the discrepancies in the MSE estimates by the ABP-derived IBI series in animal models might not be easily extended to human studies, or might be different if obtained from invasive measures at central sites or noninvasively at the finger.

\section{References}

[1] I. Constant, D. Laude, I. Murat, and J. L. Elghozi, 'Pulse rate variability is not a surrogate for heart rate variability', Clin. Sci., vol. 97, no. 4, pp. 391-397, Oct. 1999.

[2] M. Costa, A. L. Goldberger, and C.-K. Peng, 'Multiscale entropy analysis of biological signals', Phys. Rev. E, vol. 71, no. 2, p. 021906, Feb. 2005, doi: 10.1103/PhysRevE.71.021906.

[3] P. Castiglioni, G. Parati, and A. Faini, 'Information- domain analysis of cardiovascular complexity: night and day modulations of entropy and the effects of hypertension', Entropy, vol. 21, no. 6, p. 550, May 2019, doi: 10.3390/e21060550.

[4] P. Castiglioni, P. Coruzzi, M. Bini, G. Parati, and A. Faini, 'Multiscale sample entropy of cardiovascular signals: does the choice between fixed- or varying-tolerance among scales influence its evaluation and interpretation?', Entropy, vol. 19, no. 11, p. 590, Nov. 2017, doi: 10.3390/e19110590.

[5] V. V. Nikulin and T. Brismar, "Comment on "Multiscale entropy analysis of complex physiologic time series", Phys. Rev. Lett., vol. 92, no. 8, pp. 089803; author reply 089804, Feb. 2004, doi: 10.1103/PhysRevLett.92.089803.

[6] D. P. M. Dias, L. E. V. Silva, P. L. Katayama, C. a. A. Silva, H. C. Salgado, and R. Fazan, 'Correlation between $\mathrm{RR}$, inter-systolic and inter-diastolic intervals and their differences for the analysis of spontaneous heart rate variability', Physiol Meas, vol. 37, no. 7, pp. 1120-1128, 2016, doi: 10.1088/0967-3334/37/7/1120.

Address for correspondence:

Paolo Castiglioni.

IRCCS Fondazione Don C. Gnocchi

Via Capecelatro 66, I 20148, Milano, Italy.

pcastiglioni@dongnocchi.it 